\title{
On the high order asymptotic solution of certain wave equations
}

\author{
Vu Hoang Linh
}




\title{
ON THE HIGH ORDER ASYMPTOTIC SOLUTION OF CERTAIN WAVE EQUATIONS
}

\author{
VU HOANG LINH
}

[Received: August 5, 2003]

\begin{abstract}
Aвstract. The paper is devoted to the asymptotic approximation for solutions of a class of second order linear ordinary differential equations. A special form of the Liouville-Green type approximation is developed to obtain high order asymptotics. Asymptotic solutions can be constructed easily by using a recursive formula. An error estimation based on Olver's explicit error bound is proposed. The result and its applications are discussed and illustrated by some examples.
\end{abstract}

Mathematics Subject Classification: 34E05, 34E20, 65D20

Keywords: Liouville-Green (or JWKB) approximation, wave equations, high order asymptotic solutions

\section{InTRODUCtion}

$\mathrm{L}$

ET US CONSIDER the differential equation

$$
y^{\prime \prime}(x)+U(x) y(x)=0, \quad x_{0} \leq x<\infty .
$$

Assume that the real function $U(x)$ is continuous and has the asymptotics

$$
U(x) \sim x^{\sigma} \sum_{j=0}^{\infty} U_{j} x^{-j / 2}, \quad x \rightarrow \infty,
$$

where $\sigma$ and $U_{j}(j=0,1,2, \ldots)$ are given; $U_{0} \neq 0 ; \sigma$ is a nonnegative integer. The solutions of problem (1.1), (1.2) are called wave functions. In this paper, we focus on the case when $U_{0}$ is positive. The corresponding solutions are called radial wave functions. From now on, we suppose that $U_{0}$ is positive. The case with negative $U_{0}$ will be discussed in Remark 3.1. Note that no extra condition on the smoothness of $U(x)$ is needed.

We face differential equations of type (1.1), (1.2) frequently when solving problems of mathematical physics, especially those arising in quantum mechanics, see [8-11]. Asymptotic analysis of linear differential equations with irregular singularities has been discussed widely in the literature, e. g., [4, Chapter 5] and [11, Chapter 
7]. However, the construction as well as the proof of the existence of asymptotic solutions are very complicated, especially when $\sigma$ is positive. Most of the results on high order asymptotic approximations were obtained by the singular perturbation method. In the present paper, another approach is used. The asymptotic formula obtained is of the form of the well-known Liouville-Green approximation $[5,11]$.

First, let us introduce the Liouville transformation, a basic tool of the LiouvilleGreen approximation theory. Suppose that $f$ is a strictly positive, twice continuously differentiable function on the interval $[a, \infty)$, where $a \geq x_{0}$. Apply the transformation

$$
\xi=\int_{a}^{x} f(t) d t, \quad z(\xi)=\sqrt{f(x(\xi))} y(x(\xi))
$$

to equation $(1.1)$ on the interval $[a, \infty)$. The differential equation for the new function $z(\xi)$ is

$$
z^{\prime \prime}(\xi)+(1+\Phi(\xi)) z(\xi)=0, \quad 0 \leq \xi<\infty,
$$

where

$$
\Phi=\left(U-f^{2}\right) \frac{1}{f^{2}}-\left(\frac{f^{\prime}}{2 f^{3 / 2}}\right)^{\prime} \frac{1}{f^{3 / 2}}
$$

and $f^{\prime}$ denotes the derivative of $f$ with respect to variable $x$. Equation (1.4) is called the Liouville normal form of (1.1). If one could find an auxiliary function $f$ such that $\Phi \equiv 0$ on the whole interval, then one would express the general solution of the original equation by the formula

$$
y(x)=\frac{1}{\sqrt{f(x)}}\left[A \exp \left(\mathrm{i} \int_{a}^{x} f(t) d t\right)+B \exp \left(-\mathrm{i} \int_{a}^{x} f(t) d t\right)\right],
$$

where $A, B$ are arbitrary constants. Thus, a natural question arising here is whether the nonlinear second order differential equation

$$
\frac{U-f^{2}}{f^{2}}-\left(\frac{f^{\prime}}{2 f^{3 / 2}}\right)^{\prime} \frac{1}{f^{3 / 2}}=0
$$

has solution(s) on the interval $[a, \infty)$. A closed formula for a solution $f$, even if it exists, seems impossible in general. In this paper, we first show the existence of a solution of (1.5). Then, based on the condition (1.2), we construct its asymptotic formula as a power series of the variable $x$.

The paper is organized in the following way. In the next section, we summarize some basic results in the Liouville-Green approximation theory. As a consequence, a simple but low order asymptotic approximation is constructed. In Section 3 we show the existence of high order asymptotic solutions to (1.1) based on a preliminary result on an auxiliary Riccati differential equation. A method of construction as well as an error estimation are given. We conclude the paper with the discussion of applications and some illustrative examples. 


\section{The LiouviLle-Green APProximation}

The following theorem deals with the so-called Liouville-Green approximation. The statement is a reformulation of Theorem 2.2 in [11, Chapter 6] with respect to large $x$. We refer to Problem 30 in [4, Chapter 3], where the asymptotic behaviour of the solutions of (1.4) is considered.

Let $f(x)$ be a positive, twice continuously differentiable function on a given interval $[a, \infty)$ and let the error-control function be defined by equation

$$
S(x)=\frac{U(x)-f^{2}(x)}{f(x)}-\left(\frac{f^{\prime}(x)}{2 f^{3 / 2}(x)}\right)^{\prime} \frac{1}{f^{1 / 2}(x)} .
$$

Proposition 2.1. Suppose that the inequality

$$
\int_{a}^{\infty}|S(t)| d t<\infty
$$

holds. Then equation (1.1) has a pair of linearly independent solutions $y_{+}, y_{-}$such that

$$
y_{ \pm}(x)=\frac{1}{\sqrt{f(x)}} \exp \left( \pm \mathrm{i} \int_{a}^{x} f(t) d t\right)\left(1+\varepsilon_{ \pm}(x)\right), \quad x \rightarrow \infty .
$$

These solutions form a complex conjugate pair. Moreover, the estimate

$$
\left|\varepsilon_{ \pm}(x)\right| \leq \exp \left\{\int_{x}^{\infty}|S(t)| d t\right\}-1
$$

holds for the error terms $\varepsilon_{ \pm}(x)$.

Approximation (2.2) was obtained first by Liouville and Green, while the explicit error bound is due to Olver $[5,11]$.

A simple approximation of the solutions of (1.1), (1.2) is obtained as a direct consequence of Proposition 2.1. Let us introduce the function $V(x)=\sqrt{U(x)}$. It is well defined for sufficiently large arguments and has asymptotic representation

$$
V(x) \sim x^{\sigma / 2} \sum_{j=0}^{\infty} V_{j} x^{-j / 2}, \quad x \rightarrow \infty .
$$

From (1.2), the coefficients $V_{j}$ can be calculated by the recurrence formula

$$
V_{0}=\sqrt{U_{0}}, \quad V_{1}=U_{1} / 2 V_{0}, \quad V_{j}=\left(U_{j}-\sum_{k=1}^{j-1} V_{k} V_{j-k}\right) / 2 V_{0}, \quad(j=2,3, \ldots) .
$$

Let $g(x)$ be defined as the partial sum of the first $(\sigma+2)$ terms of the above asymptotic series

$$
g(x)=x^{\sigma / 2} \sum_{j=0}^{\sigma+2} V_{j} x^{-j / 2}
$$


Since $V_{0}$ is positive, so is $g(x)$ on an interval $[a, \infty)$, where $a$ is sufficiently large. Moreover, by elementary calculations, one can check that the relation

$$
\left|\frac{U(x)-g^{2}(x)}{g(x)}-\left(\frac{g^{\prime}(x)}{2 g^{3 / 2}(x)}\right)^{\prime} \frac{1}{g^{1 / 2}(x)}\right|=O\left(\frac{1}{x^{3 / 2}}\right), \quad x \rightarrow \infty,
$$

holds. This means that function $g$ satisfies condition (2.1). We obtain

Proposition 2.2. Equation (1.1), (1.2) has a pair of independent solutions $y_{+}, y_{-}$ such that

$$
y_{ \pm}(x)=\frac{1}{\sqrt{g(x)}} \exp ( \pm \mathrm{i} G(x))\left(1+O\left(\frac{1}{\sqrt{x}}\right)\right), \quad x \rightarrow \infty,
$$

where $G(x)$ is an antiderivative of $g(x)$ :

$$
G(x)=x^{\sigma / 2+1} \sum_{j=0}^{\sigma+1} \frac{2 V_{j}}{\sigma+2-j} x^{-j / 2}+V_{\sigma+2} \ln x .
$$

Another proof of this proposition can be found in [7]. A basis of the real solution space of equation (1.1), (1.2) can be created by $\operatorname{Re} y_{+}$and $\operatorname{Im} y_{+}$. Thus, it is easy to obtain an approximation of real solutions of (1.1), (1.2).

Corollary. Let $y(x)$ be an arbitrary real solution of (1.1), (1.2). Then, there exists a pair of constants $\left(r_{\infty}, \delta_{\infty}\right)$ such that

$$
y(x)=\frac{r_{\infty}}{\sqrt{g(x)}} \cos \left(G(x)+\delta_{\infty}+O\left(\frac{1}{\sqrt{x}}\right)\right), \quad x \rightarrow \infty,
$$

where $r_{\infty}>0$ is uniquely determined and $\delta_{\infty}$ is unique up to a multiple of $2 \pi$. Moreover, any pair $\left(r_{\infty}, \delta_{\infty}\right), \quad r_{\infty}>0$, defines the solution uniquely.

Approximations (2.4), (2.5) are simple, but their accuracy is low. In order to improve the accuracy of the Liouville-Green approximation, one may use the so-called higher order phase integral solution (see $[5,11,12]$ and references therein). It can be derived as follows. Suppose that $U(x)$ is positive and continuously differentiable as many times as needed. Instead of (1.1), we consider the singularly perturbed equation

$$
y^{\prime \prime}(x)+\frac{U(x)}{\epsilon^{2}} y(x)=0,
$$

where $\epsilon$ is a positive small parameter. Then, a solution $f$ of equation (1.5) is constructed as a power series of $\epsilon$

$$
f=\epsilon^{-1}\left(f_{0}+\epsilon^{2} f_{1}+\epsilon^{4} f_{2}+\ldots\right),
$$

where

$$
f_{0}=\sqrt{U}, \quad f_{1}=-\left(\frac{f_{0}^{\prime}}{2 f_{0}^{3 / 2}}\right)^{\prime} \frac{1}{2 f_{0}^{1 / 2}}, \quad \ldots
$$


If one takes the zero-order approximation, i. e., $f_{0}$ only and puts $\epsilon=1$, then the standard JWKB approximation

$$
y_{ \pm}(x) \approx U^{-1 / 4}(x) \exp \left( \pm \mathrm{i} \int_{x_{0}}^{x} \sqrt{U(t)} d t\right) .
$$

arises. The more terms are taken into consideration, the higher accuracy may be expected. However, the application of higher order approximations seems too complicated since higher derivatives of $U(x)$ are required. In addition, the integral occurring in the approximations in general, or even for the zero-order one cannot be calculated formally. This fact makes the computation of the high order approximations inconvenient and time-consuming.

In the next section, we will show that the expression on the right-hand side of (2.3) can be continued up to the asymptotic series of a solution of (1.5).

\section{High ORDER ASYMPTOTIC APPROXIMATIONS}

From now on we consider one of the solutions defined in (2.4), say $y_{+}$. One can check that the so-called radiation boundary condition

$$
\lim _{x \rightarrow \infty} \frac{x^{-\sigma / 2} y_{+}^{\prime}(x)-\mathrm{i} \sqrt{U_{0}} y_{+}(x)}{\left|x^{-\sigma / 2} y_{+}^{\prime}(x)\right|+\left|y_{+}(x)\right|}=0
$$

holds. The following crucial result can be found in $[2,6]$.

Proposition 3.1. For sufficiently large $x$, condition (3.1) can be replaced by an equivalent condition

$$
y_{+}^{\prime}(x)=R(x) y_{+}(x)
$$

Here, $R(x)$ is a solution of Cauchy problem

$$
\begin{gathered}
R^{\prime}(x)+R^{2}(x)+U(x)=0, \\
\lim _{x \rightarrow \infty} x^{-\sigma / 2} R(x)=\mathrm{i} \sqrt{U_{0}} .
\end{gathered}
$$

For large $x$, the solution of (3.2) is unique. In addition, it can be represented by an asymptotic series, namely,

$$
R(x) \sim x^{\sigma / 2} \sum_{j=0}^{\infty} \frac{R_{j}}{x^{j / 2}} \quad x \rightarrow \infty,
$$

where the coefficients $R_{j}$ can be evaluated by the recurrence formula

$$
\begin{gathered}
R_{0}=\mathrm{i} \sqrt{U_{0}}, \quad R_{1}=\frac{-U_{1}}{2 R_{0}}, \\
R_{j}= \begin{cases}\frac{-U_{j}-\sum_{s=1}^{j-1} R_{s} R_{j-s}}{2 R_{0}} & \text { if } j<\sigma+2 \\
\frac{-U_{j}-\sum_{s=1}^{j-1} R_{s} R_{j-s}+(j / 2-\sigma-1) R_{j-\sigma-2}}{2 R_{0}} & \text { if } j \geq \sigma+2,\end{cases}
\end{gathered}
$$


for $j=2,3, \ldots$.

Now, we arrive at the main results of the paper. Let $a(x), b(x)$ be the real and imaginary part of $R(x)$, respectively,

$$
a(x)=\operatorname{Re}(R(x)), \quad b(x)=\operatorname{Im}(R(x)) .
$$

Obviously, these functions are continuously differentiable and they have asymptotic series also when $x$ tends to infinity. Namely, the expansions

$$
a(x) \sim x^{\sigma / 2} \sum_{j=0}^{\infty} \frac{a_{j}}{x^{j / 2}}, \quad b(x) \sim x^{\sigma / 2} \sum_{j=0}^{\infty} \frac{b_{j}}{x^{j / 2}},
$$

are valid for large $x$, where $a_{j}=\operatorname{Re} R_{j}, b_{j}=\operatorname{Im} R_{j}, j=0,1, \ldots$ The Riccati equation in (3.2) is equivalent to the system

$$
\begin{gathered}
b^{\prime}(x)+2 a(x) b(x)=0, \\
a^{\prime}(x)+a^{2}(x)-b^{2}(x)+U(x)=0 .
\end{gathered}
$$

Theorem 3.1. For sufficiently large $x$, function $b(x)$ defined by (3.4) is positive and it is an exact solution to equation (1.5). Moreover, this solution has asymptotic expansions

$$
b(x) \sim x^{\sigma / 2} \sum_{j=0}^{\infty} \frac{b_{j}}{x^{j / 2}}, \quad x \rightarrow \infty,
$$

where the coefficients $b_{j}$ can be evaluated by the recursive formulae

$$
\begin{gathered}
b_{0}=\sqrt{U_{0}}, \quad a_{0}=0, \\
b_{j}=\frac{U_{j}+\sum_{s=1}^{j-1} a_{s} a_{j-s}-\sum_{s=1}^{j-1} b_{s} b_{j-s}-(j / 2-\sigma-1) a_{j-\sigma-2}}{2 b_{0}}, \quad j=1,2, \ldots \\
a_{j}=\frac{-2 \sum_{s=1}^{j-1} a_{s} b_{j-s}+(j / 2-\sigma-1) b_{j-\sigma-2}}{2 b_{0}}, \quad j=1,2, \ldots
\end{gathered}
$$

Here, we set $a_{s}=b_{s}=0$ for $s<0 ;\left\{a_{j}\right\}_{j=0}^{\infty}$ is considered an auxiliary sequence.

Before turning to the proof, we call attention to the fact that the first $(\sigma+3)$ terms of the asymptotic series of $b(x)$ and those of $\sqrt{U(x)}$ coincide. In general, the $(\sigma+4)$-th coefficients, however, are different.

Proof. Due to the definition, function $b(x)$ behaves like $\sqrt{U_{0}} x^{\sigma / 2}$ as $x$ tends to infinity. Therefore, there exists $x_{1}$ such that $b(x)>0$ for $x \geq x_{1}$. From the first equation in (3.5), it is clear that $b(x)$ is twice continuously differentiable. Using (3.5), one can check that

$$
\frac{U(x)-b^{2}(x)}{b^{2}(x)}-\left(\frac{b^{\prime}(x)}{2 b^{3 / 2}(x)}\right)^{\prime} \frac{1}{b^{3 / 2}(x)}=\frac{a^{\prime}(x)+a^{2}(x)-b^{2}(x)+U(x)}{b^{2}(x)}=0
$$


for $x \geq x_{1}$. It means exactly that $b(x)$ is a solution to equation (1.5). Moreover, it is unique if the condition $\lim _{x \rightarrow \infty} x^{-\sigma / 2} b(x)=\sqrt{U_{0}}$ is satisfied. Finally, we get the asymptotic series for $b(x)$ directly from (3.3) by taking the definition of $a(x)$ and $b(x)$ into account.

Due to the considerations in Section 1, the next results are obtained immediately.

Theorem 3.2. On the interval $\left[x_{1}, \infty\right)$, equation (1.1), (1.2) has a pair of solutions

$$
y_{ \pm}(x)=b^{-1 / 2}(x) \exp \left( \pm \mathrm{i} \int_{x_{1}}^{x} b(t) d t\right) .
$$

Furthermore, this solution pair is the same (up to a constant factor) as that appearing in Proposition 2.2.

Let $B(x)$ be the antiderivative of $b(x)$ defined by the asymptotics

$$
B(x) \sim x^{\sigma / 2} \sum_{\substack{j=0 \\ j \neq \sigma+2}}^{\infty} \frac{2 b_{j}}{(\sigma-j+2) x^{j / 2-1}}+b_{\sigma+2} \ln x, \quad x \rightarrow \infty .
$$

Corollary. For any real solution $y(x)$ of $(1.1),(1.2)$ defined on $\left[x_{1}, \infty\right)$, there exists a pair of constants $\left(r_{\infty}, \delta_{\infty}\right), r_{\infty}>0$, such that

$$
y(x)=\frac{r_{\infty}}{\sqrt{b(x)}} \cos \left(B(x)+\delta_{\infty}\right) .
$$

If the cosine function is replaced by the sine, we obtain another linearly independent solution. One cannot make use of the above results yet because the closed formulae of function $b(x)$ and $B(x)$ are not available. However, their asymptotic series can be calculated as we saw above. Then, by fixing an arbitrary index $N \geq \sigma+2$, there exist a pair of linearly independent solutions of problem (1.1), (1.2)

where

$$
y_{ \pm}(x)=\frac{1}{\sqrt{b_{N}(x)}} \exp \left( \pm \mathrm{i} B_{N}(x)\right)\left(1+\varepsilon_{ \pm}(x)\right)
$$

$$
b_{N}(x)=x^{\sigma / 2} \sum_{j=0}^{N} \frac{b_{j}}{x^{j / 2}}, \quad B_{N}(x)=x^{\sigma / 2} \sum_{\substack{j=0 \\ j \neq \sigma+2}}^{N} \frac{2 b_{j}}{(\sigma-j+2) x^{j / 2-1}}+b_{\sigma+2} \ln x,
$$

and

$$
\varepsilon_{ \pm}(x)=O\left(x^{(\sigma-N+1) / 2}\right), \quad x \rightarrow \infty .
$$

It is clear that the order of accuracy of approximation (3.8) can be easily increased by choosing a larger and larger index $N$. In general, by setting $N \geq \sigma+3$, we immediately get approximations of accuracy higher order than those of approximation (2.6).

For completeness, we give an estimation method for the relative error of the complex approximations based on Olver's explicit error bound. Bounds for the absolute 
and relative error of real approximations can be derived without any difficulty. For the sake of brevity, we put

$$
P(x)=\frac{3}{4}\left[b_{N}^{\prime}(x)\right]^{2}-\frac{1}{2} b_{N}^{\prime \prime}(x) b_{N}(x)-b_{N}^{2}(x)\left[b_{N}^{2}(x)-U(x)\right], \quad Q(x)=b_{N}^{3}(x) .
$$

It is easy to check that the error-control function satisfies the formula

$$
S(x)=\frac{P(x)}{Q(x)}=O\left(x^{(\sigma-N-1) / 2}\right), \quad x \rightarrow \infty
$$

and its leading coefficient is $2 b_{N+1}$. It yields immediately the asymptotic estimate for Olver's upper bound of the error function

$$
\exp \left(\int_{x}^{\infty}|S(t)| d t\right)-1 \sim \frac{2\left|b_{N+1}\right|}{N-1-\sigma} x^{(\sigma+1-N) / 2}, \quad x \rightarrow \infty .
$$

In order to get an explicit error bound, one can proceed as follows. First, we consider the case when $U(x)$ can be expressed as a finite sum, namely

$$
U(x)=x^{\sigma} \sum_{j=0}^{M} U_{j} x^{-j / 2},
$$

where $M$ is a non-negative integer. We note that this case arises frequently in applications, see [3, 8-12]. Then, both the numerator $P(x)$ and the denominator $Q(x)$ can be expressed as finite sums consisting of the powers of $x$. The following method [1] provides simple estimates for $P(x)$ and $Q(x)$. Suppose that we have to estimate the sum

$$
C(x)=\sum_{i=0}^{K} \frac{c_{i}}{x^{i / 2}}
$$

Without loss of generality we assume the leading coefficient $c_{0}>0$. Let $m$ and $n$ be the numbers of positive and negative coefficients $c_{i}, i=1,2, \ldots, K$, respectively. Take arbitrary parameters $\alpha>0$ and $\beta, 0<\beta<1$, and put

$$
x_{1}=\max _{c_{i}>0}\left\{\left(\frac{m c_{i}}{\alpha c_{0}}\right)^{2 / i}\right\} ; \quad x_{2}=\max _{c_{j}<0}\left\{\left(\frac{n c_{j}}{-\beta c_{0}}\right)^{2 / j}\right\} .
$$

Then, we obtain the estimates

$$
C(x) \leq(1+\alpha) c_{0} \text { for } x \geq x_{1}
$$

and

$$
C(x) \geq(1-\beta) c_{0} \text { for } x \geq x_{2} .
$$

Thus, based on the coefficients of $P(x)$ and $Q(x)$, we can calculate $\hat{x}_{1}, \hat{x}_{2}$ such that the estimates

and

$$
|P(x)| \leq 2(1+\alpha) b_{0}^{3}\left|b_{N+1}\right| x^{2 \sigma-(N+1) / 2}
$$

$$
|Q(x)| \geq(1-\beta)^{3} b_{0}^{3} x^{3 \sigma / 2}
$$


hold with an arbitrary pair $\alpha, \beta$ as above. Then, for $x \geq \max \left\{\hat{x}_{1}, \hat{x}_{2}\right\}$, we obtain the explicit estimate

$$
|S(x)| \leq \frac{2(1+\alpha)}{(1-\beta)^{3}}\left|b_{N+1}\right| x^{(\sigma-N-1) / 2} .
$$

Hence, for sufficiently large $x$, we obtain an explicit bound for the error terms

$$
\left|\varepsilon_{ \pm}(x)\right| \leq \exp \left(\int_{x}^{\infty}|S(t)| d t\right)-1 \lesssim \frac{4\left|b_{N+1}\right|(1+\alpha)}{(1-\beta)^{3}(N-\sigma-1)} x^{(1+\sigma-N) / 2} .
$$

When $U(x)$ has the general asymptotic expansion (1.2), we suppose that

$$
U(x)=U_{M}(x)+r(x),
$$

where $U_{M}(x)$ is as the right-hand side of (3.9) with a sufficiently large $M$ and there exists a constant $\hat{r}$ such that the estimate

$$
|r(x)| \leq \hat{r} x^{(2 \sigma-M-1) / 2}
$$

holds for the remainder term. Then, we have

$$
S(x)=\left[\frac{U_{M}(x)-b_{N}^{2}(x)}{b_{N}(x)}-\left(\frac{b_{N}^{\prime}(x)}{2 b_{N}^{3 / 2}(x)}\right)^{\prime} \frac{1}{b_{N}^{1 / 2}(x)}\right]+\frac{r(x)}{b_{N}(x)} .
$$

A procedure similar to the above gives an estimate for the first term, while the simple estimate

$$
\left|\frac{r(x)}{b_{N}(x)}\right| \leq \frac{\hat{r}}{(1-\beta) b_{0}} x^{(\sigma-M-1) / 2}
$$

holds for the second term assuming $x$ is large enough. Therefore, an explicit error bound can also be obtained in the general case.

Remark 3.1. A similar asymptotic analysis can be carried out for the exponential-type solutions of the equation

$$
y^{\prime \prime}(x)-U(x) y(x)=0, \quad x_{0} \leq x<\infty,
$$

where the same assumption on the function $U(x)$ holds as in (1.2). The new sequences $\left\{\bar{a}_{i}\right\}_{i=0}^{\infty},\left\{\bar{b}_{i}\right\}_{i=0}^{\infty}$ can be generated with the change for

$$
\begin{gathered}
\bar{b}_{0}=-\sqrt{U_{0}}, \quad \bar{a}_{0}=0, \\
\bar{b}_{j}=\frac{-U_{j}+\sum_{s=1}^{j-1} \bar{a}_{s} \bar{a}_{j-s}+\sum_{s=1}^{j-1} \bar{b}_{s} \bar{b}_{j-s}-(j / 2-\sigma-1) \bar{a}_{j-\sigma-2}}{-2 \bar{b}_{0}}, \quad j=1,2, \ldots \\
-2 \bar{b}_{0}
\end{gathered}
$$

Here, we set $\bar{a}_{s}=\bar{b}_{s}=0$ for $s<0$. Taking the finite sums $\bar{b}_{N}(x), \bar{b}_{N}(x)$ defined analogously to those in the oscillatory case, one can show that equation (3.10) has a 
pair of solutions approximated by

$$
y_{ \pm}(x)=\frac{1}{\sqrt{-b_{N}(x)}} \exp \left( \pm B_{N}(x)\right)\left(1+O\left(x^{(\sigma-N+1) / 2}\right), \quad x \rightarrow \infty .\right.
$$

Error estimates for these approximations can be obtained similarly to the oscillatory case.

\section{Applications}

Among radial wave functions occurring in practical problems, a number of wellknown special functions can be defined as solutions of differential equations of type (1.1), (1.2). Therefore, the results of Section 3 seem to be useful. For a particular solution, the value of $r_{\infty}$ is determined by some normalization condition in general. If such a condition is not prescribed or is not essential, one can fix $r_{\infty}=1$. The phase shift $\delta_{\infty}$, which plays a very important role in applications, separates definitely a particular solution from others. However, the exact formula for $\delta_{\infty}$ can only be obtained in several special cases. In most cases, $\delta_{\infty}$ should be approximated somehow, for example, see [11, Chapter 12] or [9]. For the exact values of the pair $\left\{r_{\infty}, \delta_{\infty}\right\}$ in case of some well-known radial wave functions, such as the examples below, see [3].

Example 4.1 (Airy's differential equation). First, we consider the oscillatory case

$$
y^{\prime \prime}(x)+x y(x)=0, \quad x \geq x_{0} .
$$

Using the algorithm for the computation of $\left\{b_{j}\right\}_{j=0}^{\infty}$ described in Section 3, we calculate several coefficients of the asymptotic series of $b(x)$ and get

$$
b(x) \sim x^{1 / 2}+\frac{5}{32} x^{-5 / 2}-\frac{1105}{2048} x^{-11 / 2}+\ldots, \quad x \rightarrow \infty .
$$

Consequently, we have

$$
B(x) \sim \frac{2}{3} x^{3 / 2}-\frac{10}{96} x^{-5 / 2}+\frac{2210}{18432} x^{-9 / 2}+\ldots, \quad x \rightarrow \infty .
$$

If one takes the first two terms of $b(x)$ and $B(x)$, one gets an approximate formula of the real solutions

$$
y(x) \approx \frac{r_{\infty}}{\sqrt{x^{1 / 2}+\frac{5}{32} x^{-5 / 2}}} \cos \left(\frac{2}{3} x^{3 / 2}-\frac{10}{96} x^{-5 / 2}+\delta_{\infty}\right)
$$

for large $x$. Each constant pair $\left\{r_{\infty}, \delta_{\infty}\right\}$ defines a solution uniquely. For estimating the error, we calculate

$$
P(x)=-\frac{1105}{1024} x^{-4}-\frac{25}{128^{2}} x^{-7}-\frac{5^{4}}{32^{4}} x^{-10}, \quad Q(x)=\left(x^{1 / 2}+\frac{5}{32} x^{-5 / 2}\right)^{3} .
$$

We obtain a simple estimate for the error-control function

$$
S(x) \approx-\frac{1105}{1024} x^{-11 / 2},
$$


for sufficiently large $x$.

Now, we consider the exponential case

$$
y^{\prime \prime}(x)-x y(x)=0, \quad x \geq x_{0} .
$$

We calculate the sequences $\left\{\bar{a}_{i}\right\}_{i=0}^{\infty},\left\{\bar{b}_{i}\right\}_{i=0}^{\infty}$ and obtain, for example, an approximation for exponentially decaying solutions as

$$
y(x) \approx \frac{r_{\infty}}{\sqrt{x^{1 / 2}-\frac{5}{32} x^{-5 / 2}}} \exp \left(-\frac{2}{3} x^{3 / 2}-\frac{10}{96} x^{-5 / 2}\right) .
$$

Each nonzero constant $r_{\infty}$ defines a solution uniquely. The error-control function has similar asymptotics as in the oscillatory case.

It is clear that in both the oscillatory and exponential cases, one can easily take some additional terms and obtain higher order approximate solutions.

Example 4.2 (Bessel's differential equation in normal form). Consider the equation

$$
y^{\prime \prime}(x)+\left(1-\frac{p^{2}-1 / 4}{x^{2}}\right) y(x)=0, \quad 0<x<\infty,
$$

where $p \geq 0$ is a parameter. Using the algorithm for the computation of $\left\{b_{j}\right\}_{j=0}^{\infty}$ described in Section 3, we calculate several coefficients of the asymptotic series of $b(x)$ and get

$$
b(x) \sim 1-\frac{n-1}{8 x^{2}}-\frac{(n-1)(n-25)}{128 x^{4}}+\ldots, \quad x \rightarrow \infty,
$$

where $n=4 p^{2}$. Consequently,

$$
B(x) \sim x+\frac{n-1}{8 x}+\frac{(n-1)(n-25)}{384 x^{3}}+\ldots . \quad x \rightarrow \infty .
$$

Then we obtain, for example, an approximate formula for the solutions of (4.2)

$$
y(x) \approx \frac{r_{\infty}}{\sqrt{1-\frac{n-1}{8 x^{2}}-\frac{(n-1)(n-25)}{128 x^{4}}}} \cos \left(x+\frac{n-1}{8 x}+\frac{(n-1)(n-25)}{384 x^{3}}+\delta_{\infty}\right),
$$

which holds for large $x$.

We underline that if the expressions we obtained here are expanded using the asymptotic representations of $b(x)$ and $B(x)$, then one arrives at the other frequently used asymptotic expansions of Bessel functions, which can be found in $[3,11]$.

Example 4.3 (Equation of parabolic cylinder functions). Consider the equation

$$
y^{\prime \prime}(x)+\left(\frac{x^{2}}{4}-a\right) y(x)=0, \quad 0 \leq x<\infty,
$$


where $a$ is a parameter. Analogously to the above, we get asymptotic series for $b(x)$ and $B(x)$ as follows

$$
\begin{gathered}
b(x) \sim \frac{x}{2}-\frac{a}{x}-\frac{a^{2}-3 / 4}{x^{3}}-\frac{2 a^{3}-19 a / 2}{x^{5}} \ldots, \quad x \rightarrow \infty, \\
B(x) \sim \frac{x^{2}}{4}-a \ln x+\frac{a^{2}-3 / 4}{2 x^{2}}+\frac{2 a^{3}-19 a / 2}{4 x^{4}} \ldots, \quad x \rightarrow \infty .
\end{gathered}
$$

Similarly to the previous examples, high order approximate formulae for the parabolic cylinder functions can be given.

Now let the pair $\left\{r_{\infty}, \delta_{\infty}\right\}$ of a particular solution be given. Then, for sufficiently large arguments, the solution can be approximated with high accuracy using (3.8). Even in the case when equations are more complicated than (4.1), (4.3), the coefficients $b_{j}$ are available easily by the recursive formulae (3.6), (3.7). Evaluation of the approximations can be quickly achieved and in a less complicated way since no numerical integration is necessary. This advantage is extremely important in situations when a large number of function evaluations are needed, such as in computation of integrals containing solutions.

For sufficiently large arguments, the errors of the approximation can be estimated by computing some additional terms of the asymptotic series of $b(x)$ and $B(x)$. However, one should be very careful with the use of (3.8) because of its asymptotic nature: if the argument is not large enough, increasing the index $N$ may actually lead to less accurate numerical results. To ensure the accuracy of numerical function evaluation, one should carry out the error estimation process as described in Section 3. For "small" arguments, approximation of the solutions can be obtained in other ways such as polynomial approximation or numerical integration.

For the numerical integration of the solutions of (1.1), (1.2), a variant of the socalled amplitude-phase methods (or the Prüfer methods [12]) has been proposed, see [7-9]. The application of the method to quantum mechanic problems was discussed in [8]. We do not go into detail here, just mention that a pair of auxiliary functions, the preconditioning function and the phase-shifting one, plays a very important role in the effective realization of the method [9]. One can set these functions using $b_{N}(x)$ and $B_{N}(x)$, respectively, with an appropriately chosen index $N$. The method then becomes very efficient: beside advantages gained from large admissible stepsizes in automatic integration, the interval of numerical integration can be essentially shortened. Consequently, a high accuracy of function evaluations can be easily achieved. This fact has been confirmed by numerical experiments (see, for instance, $[8,9])$. In addition to the error estimation of the approximation (3.8), another error estimation with respect to the approximation of the amplitude and phase-shift constants $\left(r_{\infty}, \delta_{\infty}\right)$ was analyzed in $[9,10]$. 


\section{REFERENCES}

[1] Abramov, A. A. AND Balla, K.: Approximate solutions, based on comparison theorems, of scalar and matrix Riccati equations in an infinite interval, Comput. Math. Math. Phys, 33 (1993), No. 1, 29-43; translated from Zh. Vychisl. Mat. Mat. Fiz., 33 (1993), No. 1, 35-51.

[2] Aвramov, A. A. and Konyukhova, N. B.: Transfer of admissible boundary conditions from a singular point for systems of linear ordinary differential equations, Sov. J. Numer. Anal. Math. Modelling, 1 (1986), No. 4, 245-265.

[3] Abramowitz, A. and Stegun, I. A.: Handbook of mathematical functions, Dover, N.Y., 1968.

[4] Coddington, E. A. And Levinson, N.: Theory of ordinary differential equations, McGraw-Hill, New York, 1955.

[5] Fedoryuk, M. V.: Asymptotic methods in analysis. In: Analysis I, Integral representation and asymptotic methods (R. V. Gamkrelidze, ed.), Springer-Verlag, 1989.

[6] Konyuknova, N. B., On admissible boundary conditions at irregular singular point for systems of linear ordinary differential equations, U.S.S.R. Comput. Math. Math. Phys., 23 (1983), No. 4 , 23-35; translated from Zh. Vychisl. Mat. Mat. Fiz., 23 (1983), No. 4, 806-824.

[7] Konyukhova, N. B., Masalovich, S. Ye., and Staroverova, I. B.: Computation of rapidly oscillating eigenfunctions of continuous spectrum and their improper integrals, Russian J. Comput. Math. Math. Phys., 35 (1995), No. 3, 287-302; translated from Zh. Vychisl. Mat. Mat. Fiz., 35 (1995), No. 3, 360-379.

[8] Konyukhova, N. B., Linh, V. H., and Staroverova, I. B.: Modifications of the method of phase functions as applied to singular problems in quantum physics, Comput. Math. Math. Phys., 39 (1999), No. 3, 468-498; translated from Zh. Vychisl. Mat. Mat. Fiz. 39 (1999), No. 3, 492-522.

[9] Lin,, V. H.: On some questions arising in numerical realization of amplitude-phase methods, J. Numerical Algorithms, 17 (1998), No. 1-2, 171-191.

[10] Linh, V. H.: Error estimates for the amplitude-phase method in the evaluation of radial wave functions, Acta Sci. Math. (Szeged), 63 (1997), No. 3-4, 657-670.

[11] Olver, F. W. J.: Asymptotics and special functions, Academic Press, New York, 1974.

[12] Pryce, J. D.: Numerical solution of Sturm-Liouville problems, Clarendon Press, Oxford, New York \& Tokyo, 1993.

\section{Author's Address}

\section{Vu Hoang Linh:}

Faculty of Mathematics, Mechanics, and Informatics, University of Natural Sciences, Vietnam

National University, 334 Nguyen Trai Str., Thanh Xuan, Hanoi

E-mail address: vhlinh@hn.vnn.vn 